Research

\title{
Acceptance and Fear of Artificial Intelligence: associations with personality in a German and a Chinese sample
}

\author{
Cornelia Sindermann ${ }^{1}$ (D) Haibo Yang ${ }^{2} \cdot$ Jon D. Elhai ${ }^{3} \cdot$ Shixin Yang ${ }^{2} \cdot$ Ling Quan $^{4} \cdot$ Mei Li $^{5} \cdot$ Christian Montag $^{1,4}$ (D)
}

Received: 6 December 2021 / Accepted: 29 December 2021

Published online: 31 January 2022

(c) The Author(s) 2022 OPEN

\begin{abstract}
Understanding individual differences in attitudes towards Artificial Intelligence (Al) is of importance, among others in system development. Against this background, we sought to investigate associations between personality and attitudes towards Al. Relations were investigated in samples from two countries-Germany and China-to find potentially replicable, cross-culturally applicable associations. In German ( $N=367, n=137$ men) and Chinese $(N=879 ; n=220$ men) online surveys, participants completed items on sociodemographic variables, the Attitudes Towards Artificial Intelligence (ATAI) scale, and the Big Five Inventory. Correlational analysis revealed among others significant positive associations between Neuroticism and fear of Al in both samples, with similar effect sizes. In addition to a significant association of acceptance of Al with gender, regression analyses revealed a small but significant positive association between Neuroticism and fear of $\mathrm{Al}$ in the German sample. In the Chinese sample, regression analyses showed positive associations of acceptance of Al with age, Openness, and Agreeableness. Fear of Al was only significantly negatively related to Agreeableness in the Chinese sample. The association of fear of Al with Neuroticism just failed to be significant in the regression model in the Chinese sample. These results reveal important insights into associations between certain personality traits and attitudes towards Al. However, given mostly small effect sizes of relations between personality and attitudes towards Al, other factors aside from personality traits seem to be of relevance to explain variance in individuals' attitudes towards Al, as well.
\end{abstract}

Keywords Artificial Intelligence · ATAI scale · Personality · Big Five

\author{
Abbreviations \\ Al Artificial Intelligence \\ ATAI Attitude Towards Artificial Intelligence \\ BFI Big Five Inventory \\ NSI Need Satisfaction Inventory
}

Cornelia Sindermann and Christian Montag equally contributed to this article

Supplementary Information The online version contains supplementary material available at https://doi.org/10.1007/s44202-022-00020-y.

Christian Montag, mail@christianmontag.de; Cornelia Sindermann, cornelia.sindermann@uni-ulm.de; Haibo Yang, yanghaibo@ tjnu.edu.cn; Jon D. Elhai, contact@jon-elhai.com; Shixin Yang, shixiny@gznu.edu.cn; Ling Quan, quanling@uestc.edu.cn; Mei Li, limei@ bucea.edu.cn | ${ }^{1}$ Ulm University, Ulm, Germany. ${ }^{2}$ Tianjin Normal University, Tianjin, China. ${ }^{3}$ University of Toledo, Toledo, USA. ${ }^{4}$ University of Electronic Science and Technology of China, Chengdu, China. ${ }^{5}$ Beijing University of Civil Engineering and Architecture, Beijing, China. 


\section{Introduction}

Artificial Intelligence (Al) is an omnipresent part of daily life for many individuals across the globe. Al is incorporated in computers, smartphones, cars, and in various machines and robots in factories (e.g., in products and services of Apple Inc. [1], BMW [2], Daimler [3], Google [4]). Recent work reveals that individuals differ in their attitudes towards Al products [5-10]. Thus, system characteristics (i.e., Al related characteristics) on their own cannot explain all the variance in individuals' attitudes towards Al. Instead, personal characteristics of users, such as personality predispositions, may explain part of the inter-individual variance in attitudes towards Al. Given the relevance of personality in an increasingly digitalized world [11], it is surprising that little is known about personality characteristics and their potential associations with attitudes towards Al. This knowledge could help to improve system development and design to support positive attitudes towards beneficial Al products. Beyond this, knowledge on individual characteristics predicting greater fear of Al can support identification of groups of individuals who might profit from special training before working in Al environments, because they are likely to fear Al without such training. Such training, in turn, might lower risks of accidents in work environments, where collaborations with Al products are becoming increasingly important. Based on these considerations, the present study aimed at examining associations between attitudes towards Al and personality traits. More specifically, these associations were investigated in a cross-cultural study including German and Chinese participants to carve out generalizable associations valid across different cultures.

Various definitions of Al exist. One definition is: "Al will be such a program which in an arbitrary world will cope not worse than a human." [12] (p. 2). Another definition comprises Al as computer systems which can independently perform actions which usually need human intelligence [13]. In line with this definition, others define Al-based computers and machines by their ability to act human-like, or to think like humans, whereas yet others define Al based on acting or thinking rationally [14]. Independent of the exact definition, Al (broadly defined) can be found in many devices and is already an inevitable part of everyday human life. For example, a multitude of Al products is prevalent on smartphones, alone: Siri on Apple smartphones (and computers) [1], Google Assistant on Android phones [4], and Al functions in apps including social media [15] or recommendation systems, to name a few. Additionally, Al is of importance in the automobile industry, medicine, and countless other sectors (e.g. [16], [17]). Kai-Fu Lee [18] even compared Al to electricity illustrating that Al represents a ground-breaking technology impacting many areas in society.

Given the emerging incorporation of $\mathrm{Al}$ in daily life, researchers are keen to understand individual differences in attitudes towards Al. Such an understanding could, among others, help building more acceptance and trust towards products with built-in Al. More acceptance and trust might be fostered by specialized training (see above). More acceptance and trust, in turn, might bring economic advantages to those societies who from early on embrace Al technologies. Clearly, embracement of Al can be fostered by a specific political agenda (as seen in China, see below).

Several studies from various fields show that there are individual differences in attitudes towards and trust in $\mathrm{Al}$ [5-10]. ${ }^{1}$ Differences in attitudes towards Al are most likely due to the various negative and positive consequences of incorporation of Al in daily life, which might differ in terms of perception between individuals: On the one hand, some experts suggest the putative replacement of certain jobs by machines, which are currently implemented by humans [19, 20]. In line with this, one common fear with regard to Al is that it will destroy jobs/job opportunities [21]. Additionally, a report from the Pew Research Center finds "[...] many have concerns about how advances in Al will affect what it means to be human, to be productive and to exercise free will" [22]. Other fears are, for example, that Al takes over control and destroys privacy [21].

On the other hand, Al also offers many advantages. Depending on the field of application, these advantages can differ. For example, Al incorporated in cars can support safer driving and prevent crashes. In the future cars might even drive completely autonomously $[2,3,23]$. Moreover, Al can help in the medical sector, among others to control health management systems or to assist surgeons [24]. Finally, Al functions incorporated in our smartphones without doubt make daily life easier. In conclusion, it does not seem surprising that individual differences in attitudes towards Al exist. While some individuals might be open to Al's advantages, others might focus more on the disadvantages associated with Al. However, it remains largely unknown whether there are personal characteristics which are associated with an overall positive or negative attitude towards Al.

\footnotetext{
1 Please note that we subsume high trust in Al under a positive attitude towards Al.
} 
Of course, $\mathrm{Al}$ is a very broad concept and many different functionalities and areas of application are known to incorporate different variants of Al. Thus, individuals' attitudes toward different Al services might vary. Nevertheless, we deem it important to investigate individuals' overall attitudes towards Al. This general attitude can be seen as the basis of more specific attitudes towards certain Al services. For example, if one's general attitude towards Al is extremely negative, it is unlikely that one has very positive attitudes to many specific Al services. This is also underlined by a study showing that the "Attitude Towards Artificial Intelligence" (ATAI) measure assessing general attitudes towards Al (and used in the present work as well) is associated with individuals' willingness to use diverse Al products such as Siri or Alexa [10].

One way of investigating individual characteristics putatively related to attitudes towards $\mathrm{Al}$ is examining personality traits. Personality can be described as "stable individual differences in cognitive, emotional and motivational aspects of mental states that result in stable behavioral action [...]" [25; $p 1]$. Among different personality taxonomies, the most widely accepted is the Five-Factor model of personality. It is based on the assumption that personality traits are hierarchically ordered and can-on the highest level of the hierarchy-be described on the basis of five broad domains. Oftentimes these five domains are called the Big Five of personality and are labelled Openness (to Experience), Conscientiousness, Extraversion, Agreeableness, and Neuroticism; easily remembered by the acronym OCEAN [26-30]. Shortly summarized, high scores in Openness describe individuals who like to try new things, are open to new ideas, experiences, and discussions. Highly conscientious individuals are more diligent, orderly, and self-disciplined. Extraversion describes the extent to which individuals are outgoing, like social contacts, and are assertive. High Agreeableness is related to being altruistic, helpful, and considerate. Finally, high scores in Neuroticism describe individuals who are anxious, worry a lot, and tend to show depressive tendencies $[26,29]$. To the best of our knowledge, personality has not been widely investigated in relation to general attitudes towards Al. An exception is one study assessing trust in Al and (among others) correlating it with Neuroticism as the only domain of the Big Five. This analysis revealed a non-significant correlation which was close to zero [31]. From our perspective, Neuroticism still could be of relevance for the present study, because not only acceptance of Al was in the focus of the study (where also trust plays a role), but also fear of Al with more neurotic persons likely fearing AI more than less neurotic persons.

Moreover, personality has already been investigated in various fields of research closely related to $\mathrm{Al}$ and attitudes towards Al. Among others, personality has been linked to components of the Technology Acceptance Model [32, 33]: perceived ease of use of a technology, perceived usefulness, attitudes, behavioral intention to use, and actual usage of the technology. However, results on these associations differ between studies. These heterogeneous results might be due to different years of investigation with technological developments in between, and/or varying technologies under investigation in different studies [34-38]. Moreover, personality has been associated with attitudes towards robots: Neuroticism of the Big Five was positively linked to negative attitudes towards robots. Extraversion was positively associated with negative attitudes towards the social influence of robots [39]. Additionally, a study found that only Agreeableness of the Big Five was positively correlated with trust in digital assistants. However, the association remained significant in a regression model only in individuals from generation Z [40]. Finally, some studies report associations between personality and trust in/agreement with automation. One of these studies reports positive associations between trust in automation and Agreeableness and Conscientiousness [41]. Another study found mild and mostly non-significant associations between personality and agreement with automated advice on a decision in a threat detection task; however, and of most importance for the present study, some negative and significant correlations of Neuroticism with the agreement measure were observed [42].

Thus, some of the Big Five domains seem to be associated with attitudes towards technology, automation, and robots, hence, putatively also Al (see also review by Matthews et al. [43]). However, it is not clear which Big Five domains are associated with attitudes towards Al, yet. As already mentioned in light of the Technology Acceptance Model, potential explanations for the inconsistent results across the aforementioned studies might be due to different technologies investigated. Based on this consideration, we deem it especially important to first examine associations between personality traits and individuals' general attitudes towards technology or-related to the present study-AI. In a second step, researchers should aim at investigating how relations of personality traits with attitudes towards Al change (or do not change) as a systematically and empirically investigated function of different features offered by various Al services. The present work seeks to contribute to the first step of this research agenda. To the best of our knowledge, the present work is the first work in this area.

Moreover, Al is a cross-cultural phenomenon incorporated in daily life of individuals across the globe. Naturally, attitudes towards Al can differ across cultures [10], as can associations between attitudes towards Al and personality. This cross-cultural difference is underlined by a study showing differences in trust in automation and in Big Five scores between samples from the US, Taiwan, and Turkey; unfortunately, however, correlations of trust in automation with the 
Big Five were not presented separately for the three samples [41]. Aside from differences, especially similar findings across samples from different cultures are of interest. Similar associations found in samples from different cultures would strengthen the assumption of universally applicable associations. Moreover, cross-cultural studies aiming at replicating findings across samples from different cultures can be viewed as an effective way dealing with the replication crisis in psychological science [44]. Based on these considerations, samples from Germany and China are investigated in the present work. These countries were chosen because of their different political views on what consequences artificial intelligence might have for society [45]. The aforementioned work by Sindermann et al. [10] revealed that on average the Chinese sample under investigation had more positive attitudes toward Al compared to the German sample under investigation. This finding might be explained by the higher incorporation of Al in everyday life in China, at least from a government's vision perspective $[46,47]$.

In conclusion, the aim of the present study is twofold: First and although not key to the present work, we revisit previous investigations on differences between Chinese and German samples in acceptance and fear towards Al. We expect higher acceptance and lower fear in the Chinese sample.

Second, the main aim of the present work is to investigate associations of individual differences in the Big Five with attitudes towards $\mathrm{Al}$ in German and Chinese samples. ${ }^{2}$ Given mostly inconsistent findings in previous research on topics closely related to personality trait associations with attitudes towards Al, we only formulate one hypothesis: Neuroticism will be negatively associated with a positive attitude toward Al. Hence, we expect Neuroticism to be negatively related to acceptance of Al and positively to fear of Al. Other associations are investigated exploratory. We expect similar Neuroticism-Al-attitude links in both the German and Chinese samples, despite potential differences in acceptance and fear levels of Al as measured with the ATAI scale.

\section{Materials and methods}

\subsection{Procedure}

The German part of the study was an online survey in the German language. It was implemented in the SurveyCoder tool $[48,49]$ and advertised via various online (e.g., social media) and offline (e.g., TV) methods. The main topic of the study was technology use, and participation was allowed from the age of 12 years. However, only individuals from age 18 are included in the present analysis (see below). Anyone with Internet access who understood the German language could participate. This led to the present German sample being a convenience sample. As an incentive, participants received feedback on their scores in some of the questionnaires, for example, the Big Five Inventory (see below). The study was approved by the local ethics committee of Ulm University, Ulm, Germany. All participants gave informed electronic consent before participation. The sample is derived from the same survey also investigating other research questions such as shown in the following papers [50,51]. Therefore, samples might in parts overlap.

The Chinese part of the study was an online survey in standard, simplified Chinese characters. The platform used for the survey was https://www.wjx.cn/. The main topics of the study were individual differences (e.g., in personality) in relation to technology use. For other publications from this project see [52,53]. Data collection took place in courses at Tianjin Normal University, Tianjin, China (a metropolitan city with around 14 million inhabitants). In addition to students taking general psychology courses who were recruited in these courses, these students also invited their friends (both on-campus and off-campus) to participate in the survey. This snowball technique was used to obtain a slightly broader sample than just psychology students. As an incentive, participants received 8-12 RMB (roughly \$1.22-1.83, November 2020). The study was approved by the local ethics committee at Tianjin Normal University, Tianjin, China. All participants gave informed electronic consent before participation.

\footnotetext{
${ }^{2}$ Originally, we also planned to investigate individual differences in satisfaction with basic needs according to Maslow's theory and associations with attitudes towards Al. However, the questionnaire used to assess differences in satisfaction with the basic needs did not show satisfactory psychometric properties in the Chinese sample. Therefore, we only present the results in the Supplementary Material.
} 


\subsection{Samples}

\subsubsection{German sample}

A total of $\mathrm{N}=431$ individuals provided data for the present study. No missing data on variables under investigation in the present work were observed. However, $n=35$ participants were excluded from analysis due to reporting being younger than 18 years or older than 100 years. Another $(n=1)$ participant was excluded due to choosing the same response option throughout at least one of the survey's pages on which variables included in the present work were presented (excluding the page with the ATAI items because choosing the same response options in all ATAl items can be reasonable given the brevity of the scale); this response pattern indicates careless responding. Finally, $n=28$ participants were excluded due to implausible answers: when asked to rank five options according to their importance for the individual participant on a scale from 1 to 5 (by assigning each option a number from $1=$ "least important" to $5=$ "most important"), they did not use each number (1-5) exactly once. Although this specific item was not relevant for the present study, we excluded these participants because wrong answers still indicate careless responding. After these data cleaning steps, a final $\mathrm{N}=367$ participants ( $n=137$ men, $n=230$ women; mean age: $M=35.26(S D=13.03)$ years, range: $18-77$ years) remained. Most of these participants reported a university/university of applied science degree as their highest educational degree $(n=189)$. The other participants reported some type of school degree as highest educational degree $(n=178)$.

\subsubsection{Chinese sample}

In total, $\mathrm{N}=1109$ Chinese individuals participated in the present study. However, $\mathrm{n}=91$ individuals who provided missing data on the variables investigated in the present work were excluded from final analysis. Moreover, $\mathrm{n}=8$ individuals were excluded because they reported an age $<18$ years or $>100$ years. Additionally, $n=41$ participants reported the same response option throughout at least one of the survey's pages on which variables included in the present work were presented (excluding the page with the ATAI items; see above). These individuals were excluded. No participant was excluded due to not correctly responding to the importance ranking (see above); Chinese participants were not allowed to respond by choosing one of the numbers (1-5) more than once given the programming of the survey. However, $n=61$ were excluded from further analysis because of being outliers in survey administration time according to the formula by Tukey [54]; please note that we only assessed participation time in the Chinese study. Additionally, $n=29$ individuals stated that their birthday was later than the beginning of 2007, which is far later than 2002. The latter year indicates age of around 18 years. These individuals were also excluded, which led to a final sample size of $N=879(n=220$ men, $n=659$ women; mean age: $M=21.00$ years, $S D=4.65$; range: $18-53$ years) Chinese participants. Most participants reported currently being undergraduate students $(n=819)$. The others stated junior/senior high school degree $(n=9)$ as highest educational degree or some type of university degree $(n=51)$.

\subsection{Measures}

\subsubsection{Attitude Towards Artificial Intelligence scale}

To assess attitudes towards artificial intelligence, the Attitude Towards Artificial Intelligence (ATAI) scale was used in German and Chinese languages, respectively; both translations have been used and published before [10]. The scale consists of five items and two scales: Acceptance ( 2 items) and Fear ( 3 items) of Artificial Intelligence. Each item is answered on an 11-point Likert-Scale from $0=$ "strongly disagree" to $10=$ "strongly agree". Internal consistency estimates using Cronbach's alpha were 0.60 for Acceptance and 0.74 for Fear in the present German sample and 0.65 and 0.55 for Acceptance and Fear in the Chinese sample.

\subsubsection{Big Five Inventory}

The Big Five of personality were assessed by means of the Big Five Inventory (BFI) $[29,55,56]$. It consists of 44 to 45 items, but the 45th item is unique to the German version. Hence, we did not take this item into account in the present analysis. The items of the BFI are answered on a 5-point Likert-scale from 1 = "very inapplicable" to 5 = "very applicable". Internal 
consistencies of the Big Five scales assessed via Cronbach's alpha were $0.78 / 0.75$ for Openness ( 10 items), $0.84 / 0.72$ for Conscientiousness ( 9 items), $0.88 / 0.69$ for Extraversion ( 8 items), $0.72 / 0.67$ for Agreeableness ( 9 items), and 0.86/0.71 for Neuroticism ( 8 items) in the German/Chinese sample. Note that in the Chinese sample, the reversed item 35 of the Openness scale ("... prefers work that is routine") showed a negative item-whole correlation against the scale without this item $(r=-0.06)$. We still used the reversed item to build the scale based on the original work [55] and because reliability was still adequate in the Chinese sample.

\subsection{Statistical analysis}

Statistical analysis was implemented in R and R Studio [57, 58].

According to the rule of thumb by Miles and Shevlin [59], normality could be assumed for the variables under investigation in the German and Chinese samples; only age showed skewness and kurtosis exceeding \pm 1 in the Chinese total, male, and female samples (and Conscientiousness showed kurtosis of 1.01 in the Chinese total sample).

First, descriptive statistics and associations of all variables of interest with age and gender were calculated using Pearson correlations and t-tests (Welch's t-tests when necessary). Of note, we decided to use Pearson correlations to investigate associations with age also in the Chinese sample given the large sample size. The analysis on associations with age and gender were of importance given the unequal gender distributions and different mean ages in the samples from Germany and China, and to include these variables (age and gender) in final analyses if necessary; see for example studies by Sindermann et al. [10] and Liang and Lee [60] reporting on associations of age and gender with attitudes towards Al. Moreover, we implemented multifactorial ANOVAs including sample (German versus Chinese), gender (men versus women) and their interaction to investigate group differences in all variables of interest.

Next, zero-order bivariate correlations between the ATAI Acceptance and Fear of AI scales and the BFI scales were investigated. Finally, linear regression analyses were performed. Specifically, four models were tested: Each of the two ATAI scales was predicted in each sample by age and gender (see significant associations in the Results section) and the Big Five scales, which were significantly correlated with the respective ATAI scale in the corresponding sample.

Additional results on associations between the ATAI scales and the Need Satisfaction Inventory scales (NSI; assessing one's satisfaction with basic need fulfillment) can be found in the Supplementary Material.

\section{Results}

\subsection{Descriptive statistics and associations with age and gender}

Significant associations with age were found for Openness $(r=0.12, p=0.025)$, Conscientiousness $(r=0.21, p<0.001)$, and Neuroticism ( $r=-0.14, p=0.005)$ in the German sample. In the Chinese sample, age was significantly correlated with Openness $(r=0.15, p<0.001)$, Conscientiousness $(r=0.24, p<0.001)$, Agreeableness $(r=0.21, p<0.001)$, Neuroticism $(r=-0.12, p<0.001)$, and the ATAI Acceptance scale $(r=0.17, p<0.001)$.

Descriptive statistics can be found in Table 1. Significant gender differences were found in Conscientiousness, Extraversion, Agreeableness, and Neuroticism in the German sample; women showed higher scores than men. Additionally, German men scored higher than German women in the ATAI Acceptance scale. Gender differences in the Chinese sample were observed in Openness, Conscientiousness, Extraversion, and Neuroticism. In all scales except Neuroticism, men showed higher scores than women (see Table 1).

Multifactorial ANOVAs (sample $x$ gender) revealed significant differences between the German and Chinese sample in ATAI Acceptance $(F(1,1242)=327.76, p<0.001)$, Openness $(F(1,1242)=65.15, p<0.001)$, Conscientiousness $(F(1,1242)=84.00, p<0.001)$, Extraversion $(F(1,1242)=33.66, p<0.001)$, and Agreeableness $(F(1,1242)=32.12$, $\mathrm{p}<0.001)$; see Table 1 for descriptive statistics. The same multifactorial ANOVAs revealed significant differences between men and women in ATAI Acceptance $(F(1,1242)=9.55, p=0.002$; men $>$ women $)$ and Neuroticism $(F(1,1242)=34.19, p<0.001$; men $<$ women). Finally, the multifactorial ANOVAs revealed significant interaction effects of sample (German versus Chinese) and gender (men versus women) on Openness $(F(1,1242)=11.21, p<0.001$ ), Conscientiousness $(F(1,1242)=23.77, p<0.001)$, Extraversion $(F(1,1242)=23.77, p<0.001)$, and Agreeableness $(F(1,1242)=8.96, p=0.003)$; see Table 1 for descriptive statistics. 
Table 1 Descriptive statistics and gender differences

\begin{tabular}{|c|c|c|c|c|c|c|c|c|c|c|c|c|c|c|}
\hline & \multicolumn{7}{|c|}{ German sample } & \multicolumn{7}{|c|}{ Chinese sample } \\
\hline & \multicolumn{2}{|c|}{$\begin{array}{l}\text { Total } \\
\text { sample } \\
(\mathrm{N}=367)\end{array}$} & \multicolumn{2}{|c|}{$\begin{array}{l}\text { Men } \\
(n=137)\end{array}$} & \multicolumn{2}{|c|}{$\begin{array}{l}\text { Women } \\
(n=230)\end{array}$} & \multirow[t]{2}{*}{ Gender differences } & \multicolumn{2}{|c|}{$\begin{array}{l}\text { Total } \\
\text { sample } \\
(n=879)\end{array}$} & \multicolumn{2}{|c|}{$\begin{array}{l}\text { Men } \\
(n=220)\end{array}$} & \multicolumn{2}{|c|}{$\begin{array}{l}\text { Women } \\
(n=659)\end{array}$} & \multirow[t]{2}{*}{ Gender differences } \\
\hline & M & SD & M & SD & M & SD & & M & SD & M & SD & M & SD & \\
\hline ATAI Acceptance & 4.26 & 1.87 & 4.62 & 1.99 & 4.05 & 1.77 & $\begin{array}{l}t(259.7)=2.81 \\
p=.005 \\
d=0.31\end{array}$ & 6.50 & 1.82 & 6.63 & 1.97 & 6.45 & 1.77 & $\begin{array}{l}t(877)=1.23 \\
p=.218 \\
d=0.10\end{array}$ \\
\hline ATAI Fear & 4.09 & 2.20 & 3.96 & 2.16 & 4.17 & 2.23 & $\begin{array}{l}t(365)=-0.87 \\
p=.384 \\
d=0.09\end{array}$ & 4.09 & 1.81 & 4.10 & 1.95 & 4.09 & 1.76 & $\begin{array}{l}t(345.2)=0.03 \\
p=.979 \\
d=0.00\end{array}$ \\
\hline Openness & 3.59 & 0.59 & 3.51 & 0.61 & 3.64 & 0.58 & $\begin{array}{l}t(365)=-1.91 \\
p=.056 \\
d=0.21\end{array}$ & 3.26 & 0.51 & 3.35 & 0.52 & 3.23 & 0.50 & $\begin{array}{l}t(877)=2.92 \\
p=.004 \\
d=0.23\end{array}$ \\
\hline Conscientiousness & 3.51 & 0.71 & 3.39 & 0.72 & 3.59 & 0.70 & $\begin{array}{l}t(365)=-2.68 \\
p=.008 \\
d=0.29\end{array}$ & 3.10 & 0.50 & 3.22 & 0.54 & 3.06 & 0.48 & $\begin{array}{l}t(341.9)=3.97 \\
p<.001 \\
d=0.33\end{array}$ \\
\hline Extraversion & 3.38 & 0.84 & 3.26 & 0.84 & 3.46 & 0.83 & $\begin{array}{l}t(365)=-2.28 \\
p=.023 \\
d=0.25\end{array}$ & 3.06 & 0.55 & 3.22 & 0.55 & 3.01 & 0.54 & $\begin{array}{l}t(877)=4.99 \\
p<.001 \\
d=0.39\end{array}$ \\
\hline Agreeableness & 3.52 & 0.56 & 3.42 & 0.57 & 3.58 & 0.55 & $\begin{array}{l}t(365)=-2.65 \\
p=.008 \\
d=0.29\end{array}$ & 3.68 & 0.48 & 3.71 & 0.52 & 3.67 & 0.47 & $\begin{array}{l}t(877)=1.12 \\
p=.265 \\
d=0.09\end{array}$ \\
\hline Neuroticism & 3.00 & 0.80 & 2.86 & 0.78 & 3.08 & 0.79 & $\begin{array}{l}t(365)=-2.53 \\
p=.012 \\
d=0.27\end{array}$ & 3.01 & 0.58 & 2.80 & 0.57 & 3.08 & 0.56 & $\begin{array}{l}t(877)=-6.40 \\
p<.001 \\
d=0.50\end{array}$ \\
\hline
\end{tabular}

Table 2 Zero-order bivariate correlations of the ATAI scales with the BFI

\begin{tabular}{llllll}
\hline & \multicolumn{2}{l}{$\begin{array}{l}\text { German sample } \\
(\mathrm{n}=367)\end{array}$} & & & $\begin{array}{l}\text { Chinese sample } \\
(\mathrm{n}=879)\end{array}$ \\
\cline { 2 - 3 } \cline { 5 - 6 } & ATAI Acceptance & ATAI Fear & & ATAI Acceptance & ATAI Fear \\
\hline Openness & $\mathrm{r}=-0.06$, & $\mathrm{r}=-0.03$, & & $\mathrm{r}=0.19$, & $\mathrm{r}=-0.02$, \\
Conscientiousness & $\mathrm{p}=.262$ & $\mathrm{p}=.539$ & & $\mathrm{p}<.001$ & $\mathrm{p}=.533$ \\
Extraversion & $\mathrm{r}=-0.03$, & $\mathrm{r}=-0.08$, & & $\mathrm{r}=0.12$, & $\mathrm{r}=-0.09$, \\
& $\mathrm{p}=.591$ & $\mathrm{p}=.114$ & & $\mathrm{p}<.001$ & $\mathrm{p}=.008$ \\
Agreeableness & $\mathrm{r}=-0.06$, & $\mathrm{r}=-0.03$, & & $\mathrm{r}=0.09$, & $\mathrm{r}=-0.10$, \\
& $\mathrm{p}=.292$ & $\mathrm{p}=.506$ & & $\mathrm{p}=.008$ & $\mathrm{p}=.004$ \\
Neuroticism & $\mathrm{r}=-0.07$, & $\mathrm{r}=-0.06$, & & $\mathrm{r}=0.17$, & $\mathrm{r}=-0.15$, \\
& $\mathrm{p}=.169$ & $\mathrm{p}=.251$ & & $\mathrm{p}<.001$ & $\mathrm{p}<.001$ \\
& $\mathrm{r}=-0.04$, & $\mathrm{r}=0.14$, & & $\mathrm{r}=-0.09$, & $\mathrm{r}=0.14$, \\
& $\mathrm{p}=.433$ & $\mathrm{p}=.006$ & $\mathrm{p}=.005$ & $\mathrm{p}<.001$ \\
\hline
\end{tabular}

Note: All associations between the ATAl Acceptance scale and the Big Five differed significantly ( $z$-scores $>2.32, p$-values $<.020$ ) between the German and Chinese samples, except associations with Neuroticism ( $z=0.86, p=.390)$. None of the associations of the ATAI Fear scale with the Big Five differed significantly between the German and Chinese sample (all z-scores $<1.54$, all $p$-values $>.125$ )

\subsection{Zero-order bivariate correlations}

As presented in Table 2, in the German sample a significant positive association between the ATAI Fear scale and Neuroticism was observed. In the Chinese sample, significant positive associations were found between the ATAI Acceptance scale and Openness, Conscientiousness, Extraversion, and Agreeableness, and a significant negative correlation with Neuroticism was observed. The ATAI Fear scale correlated significantly positively with Neuroticism and negatively with Conscientiousness, Extraversion, and Agreeableness in the Chinese sample. Effect sizes were, 
Table 3 Regression models to predict ATAI scales by sociodemographic variables and BFI scales in the German sample

\begin{tabular}{|c|c|c|c|c|c|c|}
\hline & \multicolumn{3}{|c|}{ ATAI Acceptance } & \multicolumn{3}{|l|}{ ATAI Fear } \\
\hline & $B(S E)$ & $\mathrm{t}$ & $\mathrm{p}$ & $B(S E)$ & $\mathrm{t}$ & $\mathrm{p}$ \\
\hline Intercept & $0.20(0.08)$ & 2.39 & .018 & $-0.04(0.09)$ & -0.43 & .664 \\
\hline Age & $-0.07(0.05)$ & -1.42 & .158 & $0.02(0.05)$ & 0.44 & .658 \\
\hline Gender & $-0.32(0.11)$ & -3.01 & .003 & $0.06(0.11)$ & 0.55 & .585 \\
\hline Neuroticism & & & & $0.14(0.05)$ & 2.72 & .007 \\
\hline
\end{tabular}

Note: In each model, only the BFI scale(s) which significantly correlated with the respective ATAI scale in the German sample were/was included. All variables except gender were z-standardized. Gender: $0=$ men, $1=$ women

\begin{tabular}{|c|c|c|c|c|c|c|}
\hline & \multicolumn{3}{|c|}{ ATAI Acceptance } & \multicolumn{3}{|l|}{ ATAI Fear } \\
\hline & Estimate (SE) & $\mathrm{t}$ & $\mathrm{p}$ & Estimate (SE) & $\mathrm{t}$ & $\mathrm{p}$ \\
\hline Intercept & $0.03(0.07)$ & 0.42 & .674 & $0.05(0.07)$ & 0.77 & .440 \\
\hline Age & $0.13(0.03)$ & 3.70 & $<.001$ & $-0.01(0.03)$ & -0.36 & .717 \\
\hline Gender & $-0.04(0.08)$ & -0.48 & .630 & $-0.07(0.08)$ & -0.89 & .376 \\
\hline Openness & $0.14(0.04)$ & 3.56 & $<.001$ & & & \\
\hline Conscientiousness & $-0.02(0.04)$ & -0.38 & .705 & $0.00(0.04)$ & 0.05 & .962 \\
\hline Extraversion & $-0.01(0.04)$ & -0.19 & .851 & $-0.05(0.04)$ & -1.31 & .190 \\
\hline Agreeableness & $0.11(0.04)$ & 2.76 & .006 & $-0.11(0.04)$ & -2.78 & .005 \\
\hline Neuroticism & $0.00(0.04)$ & 0.08 & .939 & $0.08(0.04)$ & 1.89 & .059 \\
\hline
\end{tabular}

Note: In each model, only the BFI scales which significantly correlated with the respective ATAI scale in the Chinese sample were included. All variables except gender were $z$-standardized. Gender: $0=$ men, $1=$ women
Table 4 Regression models to predict the ATAI scales by sociodemographic variables and the BFI scales in the Chinese sample

however, overall small [61]. Nevertheless, correlation magnitudes of the Neuroticism-Fear-of-Al link were very similar in the German and Chinese sample.

Manually correcting $\mathrm{p}$-values for multiple testing by the Holm correction procedure (for the $10 \mathrm{p}$-values/tests conducted in each sample) changed the Neuroticism finding in the German sample to a non-significant finding $(p>0.050)$. In the Chinese sample, all significant $(p<0.050)$ correlations remained significant after Holm correction.

Each personality scale which significantly correlated with the respective ATAI scale in the German or the Chinese sample based on uncorrected $p$-values was included in the final regression models in the respective sample.

\subsection{Regression models}

As can be seen in Table 3, in the German sample, the only significant predictor of the ATAI Acceptance scale was gender with higher scores in men. The ATAI Fear scale was significantly positively predicted only by Neuroticism.

As can be seen in Table 4, in the Chinese sample, the ATAI Acceptance scale was significantly positively predicted by age, Openness, and Agreeableness. The Fear scale was significantly negatively predicted only by Agreeableness. The effect of Neuroticism just failed to be significant.

\section{Discussion}

The present study investigated individual differences in personality and their relations to differences in general attitudes towards Al in a German and a Chinese sample. As mentioned in the introduction section, we wanted to replicate findings on greater acceptance of Al scores and lower fear of Al scores in the Chinese versus German sample [10]. Additionally, we investigated associations between personality traits and attitudes towards Al. We specifically expected Neuroticism to be negatively associated with acceptance of Al and positively associated with fear of Al. The investigation of remaining personality trait associations with attitudes towards Al (acceptance and fear of Al) was implemented on an exploratory basis. 
Regarding the comparison of attitudes towards Al, we replicated previous findings [10] by showing higher acceptance of $\mathrm{Al}$ in the Chinese compared to the German sample. However, we did not find significant differences in fear of Al between the two samples. Regarding higher acceptance levels in the Chinese versus German sample, one possible explanation is that the Chinese government strongly supports development of technologies where Al plays a crucial role since quite some time. For example, the so called "New Generation Artificial Intelligence Development Plan" (see [47]) aims at making China a leader in Al research. Such publicly communicated strategies among others hinting at large monetary investment into $\mathrm{Al}$ research with a consequent development plan might shape opinions by Chinese citizens towards having more optimistic views towards Al (i.e., that the economy and society might profit from this development) and explain the greater acceptance of Al in the Chinese sample of the present study compared to the German sample. We are aware of the fact that our sample is not representative. Nevertheless, we see pronounced differences in the ATAI (acceptance) measure when contrasting samples from Germany and China, which fits to the findings of Sindermann et al. [10]. However, surprisingly we did not replicate greater fear of Al in our German compared to the Chinese sample; an effect which was found by Sindermann et al. [10]. Since the samples in the present work and those of Sindermann et al. [10] do not overlap, differences in findings might be due to different sample characteristics. While both data collections in China (Sindermann et al. and the present work) have been carried out in the university context, the German sample in Sindermann et al. [10] was also recruited in a university setting, whereas the German sample in the present paper is a more diverse online-sample. Beyond that, differences between the German and Chinese samples regarding the acceptance scale of the ATAl inventory were also more pronounced compared to the differences between both samples in the fear of Al scale in Sindermann et al. [10]; hence, perhaps the differences in fear of Al between German and Chinese samples might be more negligible.

In the German sample, personality was barely associated with attitudes towards Al. Only Neuroticism exhibited a positive association with the scale assessing fear of Al. In the Chinese sample especially Openness and Agreeableness were positively related to acceptance of Al, while only Agreeableness was negatively related to fear of $\mathrm{Al}$ in the regression. Effect sizes were, generally speaking, small [61]. Moreover, it is important to note that the only association, which was found across samples, was the positive correlation between Neuroticism and fear of Al, which was of similar size in both samples. However, in the Chinese sample this association did not remain significant in the regression model including demographic variables and other Big Five domains. Given that the association between Neuroticism and fear of Al in the regression model in the Chinese sample was nevertheless close to significant, the link between Neuroticism and fear of Al can generally be interpreted as existent in our samples. This view is further supported by our directed hypothesis regarding this association as outlined at the end of the Introduction section.

As mentioned before, the association of fear of Al with Neuroticism was expected based on previous work from related fields of research [39, 42]. Moreover, and as mentioned in the Introduction, individuals scoring high in Neuroticism worry a lot, are moody, and anxious. In fact, one of the more narrower facets of Neuroticism assessed in the questionnaire applied in the present study is labelled "anxiety" [29]. Therefore, it seems reasonable that individuals scoring higher in Neuroticism tend to fear (new) technological inventions, such as those based on Al, more than individuals scoring low in Neuroticism.

Regarding the other associations between personality and attitudes towards Al, it turned out that none was replicated across the samples from Germany and China. This was mostly due to correlations close to zero in the German sample. Hence, personality seems to be barely associated with general attitudes towards Al in Germany; with the exception of Neuroticism. Therefore, one can assume that in the German sample other factors aside from personality are important to explain why someone accepts or fears AI. An example might be the overall attachment style of individuals, which has been associated with trust in Al previously [31].

Aside from personal characteristics, however, also different characteristics of the Al product and environment can influence differences in attitudes towards Al; this has already been proposed in the specific context of trusting Al [62]. Moreover, a study on trust in digital assistants reports positive associations between trust in these assistants and perceived reliability and system performance factors [40]; for another model about factors of trustworthy Al (or machine learning, specifically), please see, for example, Toreini et al. [63] or the review by Glikson and Woolley [64], which additionally takes into account different kinds of $\mathrm{Al}$ (robotic, embedded, etc.).

Aside from other factors putatively influencing attitudes towards Al, some limitations of the study need to be mentioned. First, neither the German nor Chinese sample is representative of the general German or Chinese populations. This is underlined, for example, by the skewed gender distribution or distributions of educational backgrounds in the samples. The skewed distribution of educational backgrounds is especially true for the Chinese sample, which mostly comprised students. This limits generalizability of the present findings to the general German and Chinese population. 
Also, the different sample sizes from Germany and China might pose a limitation because sample size influences significance levels. Next, given the cross-sectional data assessment it is not possible to draw final conclusions about causality of associations. Given the stability of personality traits, however, it is reasonable to assume that personality indeed influences initial attitudes towards Al and not the other way around. However, it must additionally be noted that when interacting with an Al product more often, trust (and the attitude towards Al) will clearly be based on reciprocal influences between the person and the Al product; hence, personal characteristics will clearly not be the only variables influencing trust (and attitudes towards Al; see also initial trust formation versus continuous trust development as discussed in Siau and Wang [62]). In light of this, it is not only possible that attitudes towards Al but also relations between personality and attitudes towards $\mathrm{Al}$ in the long run are influenced by other variables such as previous experience and familiarity with $\mathrm{Al}$ [31], performance, collaboration and communication, and security and privacy protection of the Al product [62]. Based on the specific findings of the present work, one can assume that daily practice and interactions with Al might have a more positive influence on more neurotic individuals and their attitudes towards to Al compared to more emotionally stable individuals. But such ideas need to be supported by empirical investigations. Another limitation of the present work is that the associations between personality traits and attitudes towards Al might differ depending on which Al product a participant thought of when filling in the questionnaire; see also review by Glikson and Woolley [64] who discuss emotional and cognitive trust in different kinds of Al products taking into account different levels of machine intelligence. As we did not specifically ask participants to think of a certain Al product, participants might have thought of various products, which might have blurred results on associations with personality on group level. Not assessing potential biasing variables (e.g., what Al products one was thinking of, familiarity with $\mathrm{Al}$, and so forth) constitutes another limitation of the present work. However, please note that ATAI scale scores have been associated with attitudes towards various Al products in German and Chinese samples when the scale was validated [10].

\section{Conclusions}

Despite the aforementioned limitations, the present study gives first important insights into personality-Al relations. While in Germany only Neuroticism exhibits a significant positive correlation with fear of Al, in the Chinese sample several personality traits are related to acceptance as well as fear of Al. However, effect sizes are overall small indicating that other variables explain additional variance in attitudes towards Al. In addition to investigating further important factors, future research should investigate whether personality is associated with attitudes towards specific Al products and whether there are differential associations with personality for different Al products based on specific functions. Finally, knowing what personal as well as Al related characteristics influence attitudes towards Al can help improving Al products to be more acceptable in the future. Although the personality associations are small, such knowledge could be used to implement personalized strategies to establish positive attitudes towards Al, when human-Al-interaction is at the heart of work processes. To our knowledge, the present work is among the first shedding light on associations between personality and general attitudes towards Al and hopefully is helpful for the growing number of scientists trying to understand human-Al relations.

Authors' contributions CS and CM planned the present study. LQ and ML translated the Need Satisfaction Inventory into Chinese language. HY, SY, and CM implemented data collection. CS analyzed the data and wrote the first draft of the manuscript. CM drafted the first version of the revision. This was critically revised by CS, HY, JDE, SY, LQ, ML, and CM. All authors read and approved the final manuscript.

Funding None.

Data availability The datasets generated during and/or analyzed during the current study are available in the Open Science Framework repository (https://osf.io/r63qs/; folder: ATAI and Personality in German and Chinese samples).

\section{Declarations}

Ethics approval and consent to participate The present research had ethics approval and participants provided informed consent.

Competing interests There is no conflict of interest.

Open Access This article is licensed under a Creative Commons Attribution 4.0 International License, which permits use, sharing, adaptation, distribution and reproduction in any medium or format, as long as you give appropriate credit to the original author(s) and the source, provide a link to the Creative Commons licence, and indicate if changes were made. The images or other third party material in this article are included 
in the article's Creative Commons licence, unless indicated otherwise in a credit line to the material. If material is not included in the article's Creative Commons licence and your intended use is not permitted by statutory regulation or exceeds the permitted use, you will need to obtain permission directly from the copyright holder. To view a copy of this licence, visit http://creativecommons.org/licenses/by/4.0/.

\section{References}

1. Apple Inc. Siri. Apple (Deutschland). 2020. https://www.apple.com/de/siri/. Accessed 19 Oct 2020.

2. BMW. Artificial Intelligence. https://www.bmwgroup.com/en/innovation/technologies-and-mobility/artificial-intelligence.html. Accessed 4 Nov 2020.

3. Daimler. Two Letters and Four Principles: How Daimler Uses Artificial Intelligence (Al). https://www.daimler.com/sustainability/data/kiguidelines.html. Accessed 11 Jan 2022.

4. Google. Google Assistant on your phone. https://assistant.google.com/intl/en_uk/platforms/phones/. Accessed 11 Jan 2022.

5. Hussain I. Attitude of university students and teachers towards instructional role of artificial intelligence. Int J Dist Educ E-Learn. 2020;5:158-77. https://doi.org/10.36261/ijdeel.v5i2.1057.

6. Krogue K. Artificial Intelligence is here to stay, but consumer trust is a must for Al in business. Forbes. 2017; https://www.forbes.com/ sites/kenkrogue/2017/09/11/artificial-intelligence-is-here-to-stay-but-consumer-trust-is-a-must-for-ai-in-business/. Accessed 19 Oct 2020.

7. Martin BAS, Jin HS, Wang D, Nguyen H, Zhan K, Wang YX. The influence of consumer anthropomorphism on attitudes towards artificial intelligence trip advisors. J Hosp Tour Manag. 2020;44:108-11. https://doi.org/10.1016/j.jhtm.2020.06.004.

8. Pinto dos Santos D, Giese D, Brodehl S, Chon SH, Staab W, Kleinert R, et al. Medical students' attitude towards artificial intelligence: a multicentre survey. Eur Radiol. 2019;29:1640-6. https://doi.org/10.1007/s00330-018-5601-1.

9. Schepman A, Rodway P. Initial validation of the general attitudes towards Artificial Intelligence Scale. Comput Hum Behav Rep. $2020 ; 1: 100014$. https://doi.org/10.1016/j.chbr.2020.100014.

10. Sindermann C, Sha P, Zhou M, Wernicke J, Schmitt HS, Li M, et al. Assessing the attitude towards artificial intelligence: Introduction of a short measure in German, Chinese, and English language. Künstliche Intelligenz. 2021;35:109-18. https://doi.org/10.1007/s13218-020-00689-0.

11. Montag C, Elhai JD. A new agenda for personality psychology in the digital age? Pers Individ Differ. 2019;147:128-34. https://doi.org/10.1016/j. paid.2019.03.045.

12. Dobrev D. A definition of artificial intelligence. 2012. arXiv.org - Computer Science - Artificial Intelligence arXiv:1210.1568. Accessed 4 Nov 2020.

13. Zhang B, Dafoe A. Artificial Intelligence: American attitudes and trends. Rochester, NY: Social Science Research Network; 2019 Jan. Report No.: ID 3312874. https://papers.ssrn.com/abstract=3312874.

14. Russell S, Norvig P. Artificial Intelligence: A Modern Approach, Global Edition. 3rd ed. Boston: Addison Wesley; 2016.

15. Marr B. 4 Mind-Blowing Ways Facebook Uses Artificial Intelligence. Forbes. 2016. https://www.forbes.com/sites/bernardmarr/2016/12/ 29/4-amazing-ways-facebook-uses-deep-learning-to-learn-everything-about-you/. Accessed 19 Oct 2020

16. Marr B. The 10 Best Examples Of How Al Is Already Used In Our Everyday Life. Forbes. 2019. https://www.forbes.com/sites/bernardmarr/ 2019/12/16/the-10-best-examples-of-how-ai-is-already-used-in-our-everyday-life/. Accessed 19 Oct 2020.

17. Mills T. Council Post:The Impact Of Artificial Intelligence In The Everyday Lives Of Consumers. Forbes. 2018. https://www.forbes.com/sites/ forbestechcouncil/2018/03/07/the-impact-of-artificial-intelligence-in-the-everyday-lives-of-consumers/. Accessed 19 Oct 2020

18. Lee K-F. AI Superpowers: China, Silicon Valley, and the New World Order. Illustrated Edition. Houghton Mifflin Harcourt; 2018.

19. Frey CB, Osborne MA. The future of employment: How susceptible are jobs to computerisation? Technol Forecast Soc Change. 2017;1 14:25480. https://doi.org/10.1016/j.techfore.2016.08.019.

20. Loesche D. Die gesellschaftlichen Kosten der digitalen Revolution. 2017. https://de.statista.com/infografik/11381/automatisierung-derarbeitswelt/. Accessed 3 Sep 2019

21. Marsden P. Sex, lies and A.I.. 2017. https://assets.website-files.com/59c269cb7333f20001b0e7c4/59d7792c6e475e0001de1a2c_Sex_lies_ and_Al-SYZYGY-Digital_Insight_Report_2017_DE.pdf

22. Anderson J, Rainie L, Luchsinger A. Artificial Intelligence and the future of humans. 2018. https://www.pewresearch.org/internet/wp-conte nt/uploads/sites/9/2018/12/PI_2018.12.10_future-of-ai_FINAL1.pdf. Accessed 11 Jan 2022.

23. Waymo. We're building the World's Most Experienced DriverTM. 2020. https://waymo.com/. Accessed 27 Nov 2020.

24. Hamet $P$, Tremblay J. Artificial intelligence in medicine. Metabolism. 2017;69:36-40. https://doi.org/10.1016/j.metabol.2017.01.011.

25. Montag C, Panksepp J. Primary emotional systems and personality: an evolutionary perspective. Front Psychol. 2017;8:464. https://doi.org/10. 3389/fpsyg.2017.00464/full.

26. Costa PT, McCrae RR. Revised NEO Personality Inventory (NEO-PI-R) and NEO Five-Factor Inventory (NEO-FFI) professional manual. Odessa, Fl: Psychological Assessment Resources; 1992.

27. Fiske DW. Consistency of the factorial structures of personality ratings from different sources. The Journal of Abnormal and Social Psychology. 1949;44:329-44. https://doi.org/10.1037/h0057198

28. Goldberg LR. An alternative "description of personality": the Big-Five factor structure. J Pers Soc Psychol. 1990;59:1216-29. https://doi.org/10. 1037/0022-3514.59.6.1216.

29. Rammstedt B, Danner D. Die Facettenstruktur des Big Five Inventory (BFI). Diagnostica. 2017;63:70-84. https://doi.org/10.1026/0012-1924/a0001 61.

30. Tupes EC, Christal RE. Recurrent personality factors based on trait ratings. J Personality. 1992;60:225-51. https://doi.org/10.1111/j.1467-6494.1992. tb00973.x.

31. Gillath O, Ai T, Branicky M, Keshmiri S, Davison R, Spaulding R. Attachment and trust in artificial intelligence. Comput Hum Behav. 2021;115:106607. https://doi.org/10.1016/j.chb.2020.106607 
32. Davis FD. A technology acceptance model for empirically testing new end-user information systems : theory and results [Thesis]. Massachusetts Institute of Technology; 1986. https://dspace.mit.edu/handle/1721.1/15192. Accessed 6 Sep 2019.

33. Davis FD. Perceived usefulness, perceived ease of use, and user acceptance of information technology. MIS Q. 1989;13:319-40. https://doi.org/ $10.2307 / 249008$.

34. Barnett T, Pearson AW, Pearson R, Kellermanns FW. Five-factor model personality traits as predictors of perceived and actual usage of technology. Eur J Inf Syst. 2015;24:374-90. https://doi.org/10.1057/ejis.2014.10.

35. Devaraj S, Easley RF, Crant JM. How does personality matter? Relating the five-factor model to technology acceptance and use. Inf Syst Res. 2008;19:93-105. https://doi.org/10.1287/isre.1070.0153.

36. McElroy JC, Hendrickson A, Townsend AM, DeMarie SM. Dispositional factors in internet use: personality versus cognitive style. MIS Q. 2007;31:80920. https://doi.org/10.2307/25148821.

37. ÖzbekV, Alnıaçık Ü, Koç F, Akkılıç ME, Kaş E. The impact of personality on technology acceptance: a study on smart phone users. Proc Soc Behav Sci. 2014;150:541-51. https://doi.org/10.1016/j.sbspro.2014.09.073.

38. Svendsen GB, Johnsen J-AK, Almås-Sørensen L, Vitters $ø$ J. Personality and technology acceptance: the influence of personality factors on the core constructs of the Technology Acceptance Model. Behav Inf Technol. 2013;32:323-34. https://doi.org/10.1080/0144929X.2011.553740.

39. Müller SL, Richert A. The Big-Five personality dimensions and attitudes towards robots: a cross sectional study. Proceedings of the 11th PErvasive Technologies Related to Assistive Environments Conference. New York, NY, USA: Association for Computing Machinery; 2018; p. 405-8. https:// doi.org/10.1145/3197768.3203178.

40. Noah B, Sethumadhavan A. Generational differences in trust in digital assistants. Proceedings of the Human Factors and Ergonomics Society Annual Meeting. SAGE Publications Inc; 2019;63:206-10. https://doi.org/10.1177/1071181319631029.

41. Chien S-Y, Sycara K, Liu J-S, Kumru A. Relation between trust attitudes toward automation, Hofstede's cultural dimensions, and Big Five personality traits. Proceedings of the Human Factors and Ergonomics Society Annual Meeting. SAGE Publications Inc; 2016;60:841-5. https://doi.org/10. $1177 / 1541931213601192$.

42. Szalma JL, Taylor GS. Individual differences in response to automation: the five factor model of personality. J Exp Psychol. 2011;17:7196. https://doi.org/10.1037/a0024170.

43. Matthews G, Hancock PA, Lin J, Panganiban AR, Reinerman-Jones LE, Szalma JL, et al. Evolution and revolution: personality research for the coming world of robots, artificial intelligence, and autonomous systems. Personality Individ Differ. 2020;169:109969. https://doi.org/ 10.1016/j.paid.2020.109969.

44. Montag C. Cross-cultural research projects as an effective solution for the replication crisis in psychology and psychiatry. Asian J Psychiatr. 2018;38:31-2. https://doi.org/10.1016/j.ajp.2018.10.003.

45. Kinkartz S. Skeptical Germany lags behind on artificial intelligence | DW | 29.12.2019 [Internet]. DW.COM. [cited 2021 Dec 21]. Available from: https://www.dw.com/en/skeptical-germany-lags-behind-on-artificial-intelligence/a-51828604.

46. Wu F, Lu C, Zhu M, Chen H, Zhu J, Yu K, et al. Towards a new generation of artificial intelligence in China. Nat Mach Intell. 2020;2:3126. https://doi.org/10.1038/s42256-020-0183-4.

47. Roberts H, Cowls J, Morley J, Taddeo M, Wang V, Floridi L. The Chinese approach to artificial intelligence: an analysis of policy, ethics, and regulation. Al \& Soc. 2021;36:59-77. https://doi.org/10.1007/s00146-020-00992-2.

48. Kannen C. ckannen Softwareentwicklung. 2018. https://ckannen.com/. Accessed 28 Sep 2020.

49. Kannen C. SurveyCoder. 2020. https://www.surveycoder.com/. Accessed 28 Sep 2020.

50. Rozgonjuk D, Sindermann C, Elhai JD, Montag C. Comparing smartphone, WhatsApp, Facebook, Instagram, and Snapchat: which platform elicits the greatest use disorder symptoms? Cyberpsychol Behav Soci Netw. 2021;24:129-34. https://doi.org/10.1089/cyber.2020.0156.

51. Sindermann C, Kuss DJ, Throuvala MA, Griffiths MD, Montag C. Should we pay for our social media/messenger applications? Preliminary data on the acceptance of an alternative to the current prevailing data business model. Front Psychol. 2020;11:1415.

52. Sindermann C, Yang H, Yang S, Elhai JD, Montag C. Willingness to accept (WTA), willingness to pay (WTP), and the WTA/WTP disparity in Chinese social media platforms: Descriptive statistics and associations with personality and social media use. Acta Psychol. 2022;223:103462.

53. Montag C, Sindermann C, Rozgonjuk D, Yang S, Elhai JD, Yang H (2021) Investigating Links Between Fear of COVID-19 Neuroticism Social Networks Use Disorder and Smartphone Use Disorder Tendencies. Frontiers in Psychology 12. https://doi.org/10.3389/fpsyg.2021.682837.

54. Tukey JW. Exploratory data analysis. 1st ed. Reading, Mass: Pearson; 1977.

55. John OP, Donahue EM, Kentle RL. The Big-Five Inventory-version 4a and 54. Berkley: Berkeley Institute of Personality and Social Research, University of California; 1991.

56. Pervin LA, John OP. Personality: theory and research (Chinese version). 1st ed. Shanghai: East China Normal University Press; 2003.

57. R Core Team. R: a language and environment for statistical computing [Internet]. R Foundation for Statistical Computing; 2018. https:// www.R-project.org/

58. RStudio Team. RStudio: integrated development for R [Internet]. Boston, MA: RStudio, PBC; 2020. http://www.rstudio.com/.

59. Miles J, Shevlin M. Applying regression and correlation: a guide for students and researchers. London: SAGE Publications; 2001.

60. Liang $Y$, Lee $S A$. Fear of autonomous robots and artificial intelligence: Evidence from national representative data with probability sampling. Int J of Soc Robotics. 2017;9:379-84. https://doi.org/10.1007/s12369-017-0401-3.

61. Cohen J. A power primer. Psychol Bull. 1992;112:155-9. https://doi.org/10.1037/0033-2909.112.1.155.

62. Siau K, Wang W. Building trust in artificial intelligence, machine learning, and robotics. Cutter Bus Technol J. 2018;31:47-53.

63. Toreini E, Aitken M, Coopamootoo K, Elliott K, Zelaya CG, van Moorsel A. The relationship between trust in Al and trustworthy machine learning technologies. Proceedings of the 2020 Conference on Fairness, Accountability, and Transparency. New York, NY, USA: Association for Computing Machinery; 2020 [cited 2020 Nov 5]. p. 272-83. https://doi.org/10.1145/3351095.3372834.

64. Glikson E, Woolley AW. Human trust in Artificial Intelligence: review of empirical research. ANNALS. 2020;14:627-60. https://doi.org/10. 5465/annals.2018.0057.

Publisher's Note Springer Nature remains neutral with regard to jurisdictional claims in published maps and institutional affiliations. 